\title{
A Generative Deterministic Model for Digital Mobile Fading Channels
}

\author{
Cheng-Xiang Wang, Student Member, IEEE, and Matthias Pätzold, Senior Member, IEEE
}

\begin{abstract}
In this letter, a novel generative deterministic model (GDM) is proposed for the simulation of error processes encountered in digital mobile fading channels. The proposed GDM is simply a properly parameterized deterministic process followed by a threshold detector and two mappers. Simulation results show that this generative model enables us to match very closely any given error-free run distribution (EFRD) and error cluster distribution (ECD) of the underlying descriptive model.
\end{abstract}

Index Terms-Burst error statistics, deterministic processes, digital mobile fading channels, generative models.

\section{INTRODUCTION}

D IGITAL mobile fading channels often exhibit statistical dependencies among errors. This results in the fact that errors tend to occur in clusters or bursts. The study of the underlying bursty error process is a prerequisite for the design and performance evaluation of wireless communication protocols as well as coding systems. Error models have, therefore, been developed, basing on either descriptive approaches [1] or generative approaches [2].

In the literature, a number of generative models have been presented based on finite [2], [3] or infinite [2] state Markov chains or hidden Markov chains [4]. Among these models, simplified Frichman's models (SFMs) with only one error state [3] have received wide applications [5]. Recently, an initial attempt was carried out in [6] to utilize deterministic processes [7] for the development of generative models in digital Rayleigh fading channels. The deterministic process based generative model (DPBGM) [6] was shown to be a promising alternative to Markov models. However, the fitting to the desired error-free run distribution (EFRD) by using the DPBGM is not as good as the result obtained from a SFM with 6 states [6]. In this letter, an improved DPBGM is proposed, which enables us to nearly perfectly match any given EFRD and error cluster distribution (ECD) of the underlying descriptive model.

\section{DESCRIPTIVE STATISTICS}

An error sequence is represented by a binary sequence of ones and zeros, where " 1 " and " 0 " denote error bits and correct bits,

Manuscript received August 25, 2003. The associate editor coordinating the review of this letter and approving it for publication was Prof. F. Jondral. This work was supported by the Norwegian Research Council, Ericsson AS Grimstad, and Telenor FoU Agder under the project 144219/221:"Mobile Communication-The Mobile Student".

The authors are with the Department of Information and Communication Technology, Faculty of Engineering and Science, Agder University College, N-4876 Grimstad, Norway (e-mail: cheng.wang@hia.no; matthias.paetzold@hia.no).

Digital Object Identifier 10.1109/LCOMM.2004.825718 respectively. A gap is defined as a string of consecutive zeros between two ones, having the length equal to the number of zeros [5]. An error cluster is a region where the errors occur consecutively. The length of an error cluster equals the number of ones. In this letter, only the following two burst error statistics are to be considered. The first frequently employed statistic is the EFRD $P\left(0^{m_{g}} / 1\right)$, which is defined as the probability that an error is followed by at least $m_{g}$ error-free bits. The second one is the $\operatorname{ECD} P\left(1^{m_{c}} / 0\right)$, which is the probability that a correct bit is followed by $m_{c}$ or more consecutive bits in error [3].

To avoid a bit-by-bit analysis of the error sequence, a sensible way of recording error data is to list the successive gap lengths and error cluster lengths. From such records, the inference of the EFRD and the ECD is straightforward. Consequently, a gap recorder $\mathbf{G}_{\text {rec }}$ and an error cluster recorder $\mathbf{C}_{\text {rec }}$ are obtained. For the derivation of the generative model in Section III, it is convenient to further define the following quantities:

1) $N_{t}$ : the total length of the target error sequence.

2) $\mathcal{N}_{\mathcal{G}}:$ the total number of gaps.

3) $\mathcal{N}_{\mathcal{C}}:$ the total number of error clusters.

4) $N_{G}\left(m_{g}\right)$ : the number of gaps of length $m_{g}$ in $\mathbf{G}_{\text {rec }}$. Apparently, $\sum_{m_{g}=m_{G 1}}^{m_{G 2}} N_{G}\left(m_{g}\right)=\mathcal{N}_{\mathcal{G}}$ holds, where $m_{G 1}$ and $m_{G 2}$ denote the minimum value and the maximum value in $\mathbf{G}_{\text {rec }}$, respectively.

5) $N_{C}\left(m_{c}\right)$ : the number of error clusters of length $m_{c}$ in $\mathrm{C}_{\text {rec }}$. Similarly, $\sum_{m_{c}=m_{C 1}}^{m_{C 2}} N_{C}\left(m_{c}\right)=\mathcal{N}_{\mathcal{C}}$ holds, where $m_{C 1}$ and $m_{C 2}$ denote the minimum value and the maximum value in $\mathbf{C}_{\mathrm{rec}}$, respectively.

\section{Generative Deterministic Model}

It is well known that the statistics of burst errors can be estimated from the level-crossing statistics of the fading envelope process. This suggests the possibility that generative models can be developed from fading processes. The idea of the proposed generative model is to derive directly from a deterministic envelope process a gap length generator and an error cluster length generator. The employed deterministic process $\tilde{\zeta}(t)$ is properly parameterized and sampled with a certain sampling interval $T_{A}$. The sampled deterministic process $\tilde{\zeta}\left(k T_{A}\right)$, where $k \in \mathbf{Z}$ with $\mathbf{Z}$ as the set of integers, is then followed by a threshold detector. Error clusters are produced if the level of $\tilde{\zeta}\left(k T_{A}\right)$ falls below a given threshold $r_{t h}$. The lengths of the generated error clusters equal the numbers of samples in corresponding fading intervals of $\tilde{\zeta}\left(k T_{A}\right)$. On the other hand, gaps are generated if the level of $\tilde{\zeta}\left(k T_{A}\right)$ is above $r_{t h}$. The gap lengths equal the numbers of samples in corresponding inter-fade intervals of $\tilde{\zeta}\left(k T_{A}\right)$. Consequently, an error cluster length generator $\widetilde{\mathbf{C}}_{\text {rec }}$ and a gap 
length generator $\widetilde{\mathbf{G}}_{\text {rec }}$ are obtained. For the generative model, we use similar notations to those in Section II by simply putting the tilde sign on all affected symbols, i.e., we write $\tilde{m}_{C 1}, \tilde{\mathcal{N}}_{\mathcal{C}}$, $\tilde{N}_{G}\left(m_{g}\right)$, etc.

\section{A. The Level-Crossing Rate (LCR) Fitting}

The parameters of the deterministic process are determined by fitting the LCR at the chosen threshold to the desired occurrence rate of error clusters. Let us consider the following continuous-time deterministic process [7]

$$
\tilde{\zeta}(t)=\left|\tilde{\mu}_{1}(t)+j \tilde{\mu}_{2}(t)\right|
$$

where

$$
\tilde{\mu}_{i}(t)=\sum_{n=1}^{N_{i}} c_{i, n} \cos \left(2 \pi f_{i, n} t+\theta_{i, n}\right), \quad i=1,2 .
$$

In (2), $N_{i}$ defines the number of sinusoids, $c_{i, n}, f_{i, n}$, and $\theta_{i, n}$ are called the gains, the frequencies, and the phases, respectively. By using the method of exact Doppler spread (MEDS) [7], the phases $\theta_{i, n}$ are equated with the realizations of a random generator uniformly distributed over $(0$, $2 \pi]$, while $c_{i, n}$ and $f_{i, n}$ are given by $c_{i, n}=\sigma_{0} \sqrt{2 / N_{i}}$ and $f_{i, n}=f_{\max } \sin \left[\pi(n-1 / 2) /\left(2 N_{i}\right)\right]$, respectively. Here, $\sigma_{0}$ is the square root of the mean power of $\tilde{\mu}_{i}(t)$ and $f_{\max }$ represents the maximum Doppler frequency.

When using the MEDS, it has been shown in [7] that the LCR $\tilde{N}_{\zeta}(r)$ of $\tilde{\zeta}(t)$ is very close to the LCR $N_{\zeta}(r)$ of a Rayleigh process, which is given by

$$
N_{\zeta}(r)=\sqrt{\frac{\beta}{2 \pi}} p_{\zeta}(r), \quad r \geq 0
$$

where $\beta=2\left(\pi \sigma_{0} f_{\max }\right)^{2}$ and $p_{\zeta}(r)=\left(r / \sigma_{0}^{2}\right)$ - $\exp \left[-r^{2} /\left(2 \sigma_{0}^{2}\right)\right], r \geq 0$, denotes the Rayleigh distribution. The task at hand is to find a proper parameter vector $\Psi=\left(N_{1}, N_{2}, \sigma_{0}, r_{t h}, f_{\max }\right)$ in order to fit the LCR $\tilde{N}_{\zeta}(r) \approx N_{\zeta}(r)$ of $\tilde{\zeta}(t)$ at $r=r_{t h}$ to the given occurrence rate $R_{C}=\mathcal{N}_{\mathcal{C}} / T_{t}$ of error clusters. Here, $T_{t}$ denotes the total transmission time of the reference transmission system, from which the target error sequence of length $N_{t}$ is obtained. To solve the problem, we first choose reasonable values for $N_{1}$, $N_{2}, \sigma_{0}$, and $r_{t h}$, e.g., $N_{1}=9, N_{2}=10, \sigma_{0}=0.1$, and $r_{t h}=0.005$. Then, performing $N_{\zeta}\left(r_{t h}\right)=R_{C}, f_{\max }$ can be calculated according to $f_{\max }=\mathcal{N}_{\mathcal{C}} / \sqrt{\pi} \sigma_{0} T_{t} p_{\zeta}\left(r_{t h}\right)$.

\section{B. The Mapping Systems}

In general, the numbers of samples located in successive fading intervals and inter-fade intervals of $\tilde{\zeta}\left(k T_{A}\right)$ are not suitable to directly generate an acceptable ECD and EFRD, respectively. Two mapping systems are therefore introduced, which map the lengths of the generated error clusters and gaps to the specified lengths, as explained subsequently. Let us first define the following useful quantities: $\mathcal{N}_{\mathcal{G M}}=\left\lfloor\left(\tilde{N}_{t} / N_{t}\right) \mathcal{N}_{\mathcal{G}}\right\rfloor$, $\mathcal{N}_{\mathcal{C M}}=\left\lfloor\left(\tilde{N}_{t} / N_{t}\right) \mathcal{N}_{\mathcal{C}}\right\rfloor, N_{G R}\left(m_{g}\right)=\left(\tilde{N}_{t} / N_{t}\right) N_{G}\left(m_{g}\right)$, and $N_{C R}\left(m_{c}\right)=\left(\tilde{N}_{t} / N_{t}\right) N_{C}\left(m_{c}\right)$. Here, $\tilde{N}_{t}$ denotes the required length of the generated error sequence and $\lfloor x\rfloor$ represents the nearest integer to $x$ toward minus infinity. The necessary sim- ulation time $\tilde{T}_{t}$ for the deterministic process can be calculated according to $\tilde{T}_{t}=\left(\mathcal{N}_{\mathcal{C M}} / \mathcal{N}_{\mathcal{C}}\right) T_{t}$.

The idea of the mapping systems is to modify $\widetilde{\mathbf{G}}_{\text {rec }}$ and $\widetilde{\mathbf{C}}_{\text {rec }}$ in such a way that $\tilde{N}_{G}\left(m_{g}\right)=N_{G M}\left(m_{g}\right)$ and $\tilde{N}_{C}\left(m_{c}\right)=$ $N_{C M}\left(m_{c}\right)$ hold, respectively, where

$$
\begin{aligned}
& N_{G M}\left(m_{g}\right) \\
& \quad= \begin{cases}\left\lfloor N_{G R}\left(m_{g}\right)\right\rfloor, & \text { if } N_{G R}\left(m_{g}\right)-\left\lfloor N_{G R}\left(m_{g}\right)\right\rfloor<\mu_{g} \\
\left\lfloor N_{G R}\left(m_{g}\right)\right\rfloor+1, & \text { if } N_{G R}\left(m_{g}\right)-\left\lfloor N_{G R}\left(m_{g}\right)\right\rfloor \geq \mu_{g}\end{cases}
\end{aligned}
$$

$$
\begin{aligned}
& N_{C M}\left(m_{c}\right) \\
& \quad= \begin{cases}\left\lfloor N_{C R}\left(m_{c}\right)\right\rfloor, & \text { if } N_{C R}\left(m_{c}\right)-\left\lfloor N_{C R}\left(m_{c}\right)\right\rfloor<\mu_{c} \\
\left\lfloor N_{C R}\left(m_{c}\right)\right\rfloor+1, & \text { if } N_{C R}\left(m_{c}\right)-\left\lfloor N_{C R}\left(m_{c}\right)\right\rfloor \geq \mu_{c} .\end{cases}
\end{aligned}
$$

Here, $\mu_{g}$ and $\mu_{c}$ are real numbers located in the interval $(0,1)$, which have to be chosen properly in order to fulfill $\sum_{m_{g}=m_{G 1}}^{m_{G 2}} N_{G M}\left(m_{g}\right)=\mathcal{N}_{\mathcal{G} \mathcal{M}}$ and $\sum_{m_{c}=m_{C 1}}^{m_{C 2}} N_{C M}\left(m_{g}\right)=$ $\mathcal{N}_{\mathcal{C} M}$, respectively. Note that the resulting EFRD $\tilde{P}\left(0^{m_{g}} / 1\right)$ will be close to the desired EFRD $P\left(0^{m_{g}} / 1\right)$, since $\tilde{N}_{G}\left(m_{g}\right)$ is almost proportional to $N_{G}\left(m_{g}\right)$. Also, the resulting ECD $\tilde{P}\left(1^{m_{c}} / 0\right)$ will match well the desired one $P\left(1^{m_{c}} / 0\right)$. Next, we will only concentrate on the procedure of properly modifying $\widetilde{\mathbf{C}}_{\text {rec }}$. The same procedure applies also to $\widetilde{\mathbf{G}}_{\text {rec }}$. For each error cluster length value $m_{c}\left(m_{C 1} \leq m_{c} \leq m_{C 2}\right)$, we first find the corresponding values $\ell_{m_{c}}^{1}$ and $\ell_{m_{c}}^{2}\left(\tilde{m}_{C 1} \leq \ell_{m_{c}}^{1}, \ell_{m_{c}}^{2} \leq \tilde{m}_{C 2}\right)$ in $\widetilde{\mathbf{C}}_{\text {rec }}$ to satisfy the following conditions: $\sum_{k=\ell_{m_{c}}^{1}}^{\ell_{m_{c}}^{2}-1} \tilde{N}_{C}(k)<$ $N_{C M}\left(m_{c}\right)$ and $\sum_{k=\ell_{m_{c}}^{1}}^{\ell_{m_{c}}^{2}} \tilde{N}_{C}(k) \geq N_{C M}\left(m_{c}\right)$. Let us define $N_{\ell_{m_{c}}^{2}}=N_{C M}\left(m_{c}\right)-\sum_{k=\ell_{m_{c}}^{1}}^{\ell_{m_{c}}^{2}-1} \tilde{N}_{C}(k)$. Clearly, $\sum_{k=\ell_{m_{c}}}^{\ell_{m_{c}}^{2}-1} \tilde{N}_{C}(k)+N_{\ell_{m_{c}}^{2}}=N_{C M}\left(m_{c}\right)$ holds. This indicates that if we map all error cluster lengths between $\ell_{m_{c}}^{1}$ and $\ell_{m_{c}}^{2}-1$ and $N_{\ell_{m_{c}}^{2}}$ error cluster lengths of $\ell_{m_{c}}^{2}$ in $\widetilde{\mathbf{C}}_{\text {rec }}$ to $m_{c}$, then $\tilde{N}_{C}\left(m_{c}\right)=N_{C M}\left(m_{c}\right)$ is satisfied. In summary, the mapping system for the error cluster length generator works as follows: if $k\left(\ell_{m_{c}}^{1} \leq k<\ell_{m_{c}}^{2}-1\right)$ samples of the deterministic process are observed in a fading interval, a mapping $k \rightarrow m_{c}$ is first performed and then an error cluster with length $m_{c}$ is generated. The resulting error sequence is simply the combination of consecutively generated gaps and error clusters.

Due to the fact that the proposed error generation mechanism does not require any random generators, the obtained generative model is completely deterministic. This motivates us to call it generative deterministic model (GDM). We stress that, although the simulation set-up phase (determining the parameter vector and performing the mappings) of the GDM requires relatively long time, the simulation run phase (generation of the error sequence) is fast. Therefore, the proposed GDM can be considered as a fast error process simulator, since it determines directly gap and cluster lengths instead of bit sequences.

\section{Simulation Results AND Discussion}

The adopted reference transmission system is a coherent QPSK system with a Rayleigh fading channel [6]. The transmission rate was set to be $F_{s}=1 / T_{s}=144 \mathrm{~kb} / \mathrm{s}$. A signal-to-noise 


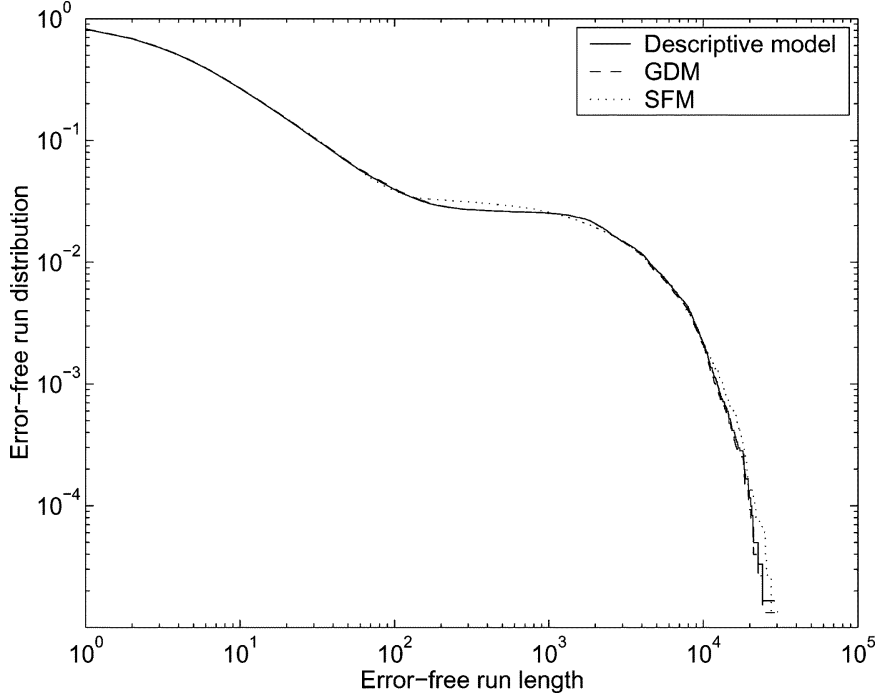

Fig. 1. EFRDs of the generative models and the descriptive model.

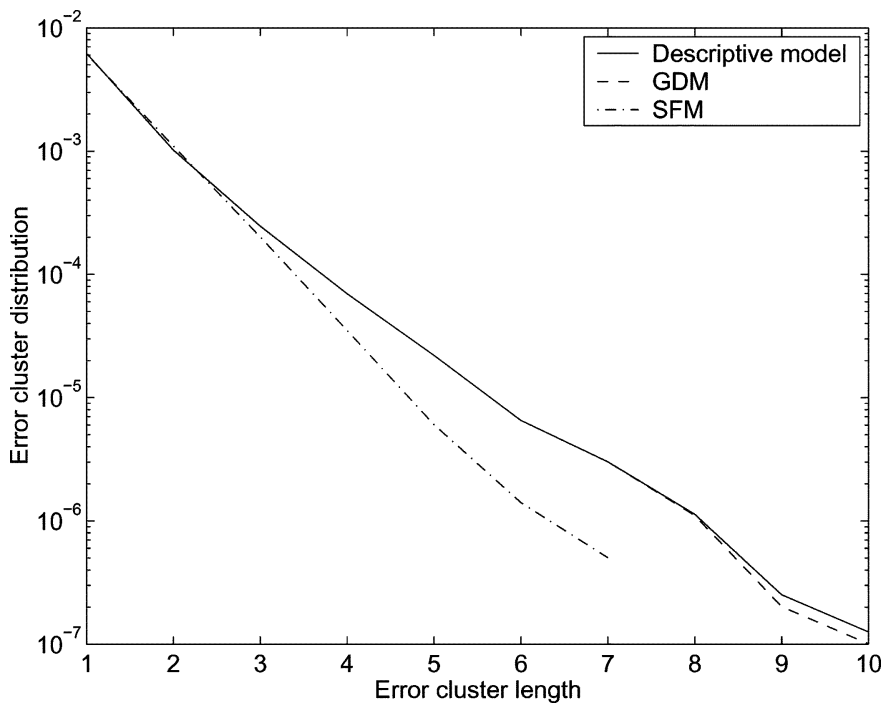

Fig. 2. ECDs of the generative models and the descriptive model.

ratio of $15 \mathrm{~dB}$ was selected for the generation of the target error sequence of length $N_{t}=8 \times 10^{6}$. This corresponds to a bit error probability of $7.5341 \times 10^{-3}$. The total transmission time is $T_{t} \approx 55.6 \mathrm{~s}$. Altogether $\mathcal{N}_{\mathcal{C}}=49379$ error clusters and $\mathcal{N}_{\mathcal{G}}=49378$ gaps were obtained.

The procedure described in Section III is applied here for obtaining the GDM. The chosen parameter vector for the deterministic process was $\Psi=(9,10,0.1,0.005,10042 \mathrm{~Hz})$. Other quantities were determined as follows: $\tilde{N}_{t}=10 \times 10^{6}, \tilde{T}_{t}=$ $69.4436 \mathrm{~s}, T_{A}=T_{s} / 24, \mu_{g}=0.25745$, and $\mu_{c}=0.75$. Figs. 1 and 2 show the EFRDs and the ECDs of the GDM, a SFM with six states [6], and the descriptive model, respectively. As expected, the near perfect match is observed in both curves for the GDM. The SFM enables a very good approximation to the EFRD of the descriptive model. However, the SFM fails to capture the feature of the ECD with good accuracy. Both generative models require relatively long time in the simulation setup phase, but the simulation run phase of the GDM is much faster than that of the SFM.

\section{CONCLUSION}

This letter proposes a novel generative model, which is simply a deterministic process followed by a threshold detector and two mapping systems. The merit of the proposed GDM lies on the fast generation of error sequences and its ability to fit nearly perfectly any given EFRD and ECD of the underlying descriptive model.

\section{REFERENCES}

[1] P. M. Crespo, R. M. Pelz, and J. Cosmas, "Channel error profiles for DECT," Proc. IEE-Commun., vol. 141, no. 6, pp. 413-420, Dec. 1994.

[2] L. N. Kanal and A. R. K. Sastry, "Models for channels with memory and their applications to error control," Proc. IEEE, vol. 66, pp. 724-744, July 1978.

[3] B. D. Frichman, "A binary channel characterization using partitioned Markov chains," IEEE Trans. Inform. Theory, vol. 13, pp. 221-227, Apr. 1967.

[4] W. Turin, Digital Transmission Systems: Performance Analysis and Modeling. New York: McGraw-Hill, 1999.

[5] F. Swarts and H. C. Ferreira, "Markov characterization of digital fading mobile VHF channels," IEEE Trans. Veh. Technol., vol. 43, pp. 977-985, Nov. 1994.

[6] C. X. Wang and M. Pätzold, "A novel generative model for burst error characterization in Rayleigh fading channels," in Proc. IEEE PIMRC'03, Beijing, China, Sept. 7-10, 2003, pp. 960-964.

[7] M. Pätzold, Mobile Fading Channels. Chichester, U.K.: Wiley, 2002. 